\title{
Research Paper \\ Mobile Access and Sexting Prevalence in High School Students in Rafsanjan City, Iran in 2015
}

\author{
Zohreh Ghorashi ${ }^{1} \odot$, "Marzeyeh Loripoor ${ }^{1} \odot$, Seyede Maryam Lotfipur-Rafsanjani ${ }^{2} \odot$
}

1. PhD. in Reproductive Health, Assistant Professor, Department of Midwifery, School of Nursing and Midwifery, Geriatric Care Research Center, Rafsanjan University of Medical Sciences, Rafsanjan, Iran.

2. MSc. in Maternal and Child Health, Departments of Midwifery, School of Nursing and Midwifery, Geriatric Care Research Center, Rafsanjan University of Medical Sciences, Rafsanjan Iran.

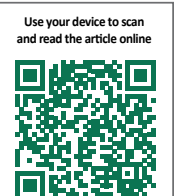

CFtation Ghorashi Z, Loripoor M, Lotfipur-Rafsanjani SM. [Mobile Access and Sexting Prevalence in High School Students in Rafsanjan City, Iran in 2015 (Persian)]. Iranian Journal of Psychiatry and Clinical Psychology. 2019; 24(4):416-425. http:// dx.doi.org/10.32598/ijpcp.24.4.416

http://dx.doi.org/10.32598/ijpcp.24.4.416

Received: 21 Sep 2017

Accepted: 01 Aug 2018

Available Online: 01 Jan 2019

Keywords:

Students, Text mes-

saging, Sexting

\begin{abstract}
A B S TRACT
Objectives Advances in the electronic communication technologies, despite a lot of benefits, have exposed the youth to some threats such as sexting. This study aimed to determine the youth access to mobile phone and the prevalence of sexting in high school students in Rafsanjan City, Iran.

Methods The study was a cross-sectional local survey of 944 high school students in Rafsanjan using stratified cluster sampling method. The survey tool was a questionnaire of texting and sexting quantity and quality. The obtained data entered the SPSS V. 16 and analyzed using Chi square and t-test.

Results Out of 994 samples, $10.9 \%$ did not access to mobile phone. About $62.7 \%$ of samples had smart phones and internet access via mobile phones. In last six months, $34.7 \%$ of samples had sent sext and $15.7 \%$ had received sext. There was a statistical significant relationship between sending $(P<0.0001)$ and receiving sext $(\mathrm{P}<0.0001)$ and the total number of texting per day.

Conclusion Iranian youths have a wide access to mobile phones and sexting is prevalent among them. Such situation would expose them to threats and dangers in sexual interactions and calls for parents' supervision and plan for parenting education.
\end{abstract}

\section{Extended Abstract}

\section{Introduction}

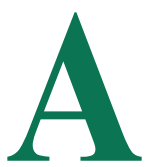

dvances in the electronic communication technologies, despite a lot of benefits, have exposed the youth to some threats such as sexting. Among American teenagers aged $12-13$ years, $68 \%$ have mobile phones and $23 \%$ smart phones and their most common usage is sending and receiving SMS, so that the average SMS transmission among them could rise up to 60 per day. Teenagers may be at particular risks and serious harms re- lated to these new technologies. In particular, using a mobile phone to send or receive naked or half-naked or sexually explicit content in recent years has got public health attention.

Sexting for the first time entered English language in 2011 and officially recorded in the Oxford Dictionary. In Iran, the use of mobile phones among teenagers is high. The average hours of mobile phone use among high school students in Tehran was 5.99 hours per week, and $1.8 \%$ of users use mobile phones for more than 16 hours a week. Of mobile phone owners, $17.9 \%$ sent over 101 text messages per week and $23.1 \%$ received over 101 text messages per week. The content of these messages in $10.7 \%$ of cases was sexual

* Corresponding Author:

Marzeyeh Loripoor, PhD.

Address: Department of Midwifery, School of Nursing and Midwifery, Rafsanjan University of Medical Sciences, Rafsanjan, Iran.

Tel: +98 (34) 34255900

E-mail: marzeyehloripoor@yahoo.com 
contents or images. Studies revealed teenagers who do sexting are more likely to become victims of siberian bullying, depression or suicide. Also, sexting was associated with vaginal, anal and oral sex, as well as unprotected sex in adolescents. This study aimed to determine the youth access to mobile phone and the prevalence of sexting in high school students in Rafsanjan.

\section{Method}

The study was a cross-sectional local survey of 944 female and male high school students in Rafsanjan City, Iran. Inclusion criteria consisted of being Iranian and under the age of 18. The presence of a known psychological disorder, as well as unwillingness to participate in research at any time, led to the exclusion from the study. All high schools in Rafsanjan were considered as research environment. We used cluster sampling method in 16 public schools, 6 special schools, 13 technical schools and 11 private schools, with a total of 10000 students in Rafsanjan City. In each category for girls and boys, a special school, a public school, a technical, and a private school were randomly selected. Calculating the sample size in each school was based on the number of students in that school and with the proportion of $10 \%$ of the total number of school students.

The questionnaires were completed by the study samples. The counselors or school deputies who had previously been informed about the appropriate sampling method collaborated in data gathering. Samples were allowed to complete the questionnaires in a private environment. To protect the privacy, the questionnaires after being completed by students, were dropped in a box. Before delivering the questionnaire, the students were assured of the confidentiality of their information. The survey tool was a questionnaire of texting and sexting quantity and quality consisted of demographic characteristics, information about having a mobile phone and how to use it to send messages, access the Internet and social networks, and sexting. The present questionnaire was inspired by similar research studies. The obtained data were entered the SPSS version 16 and analyzed using Chi square and t-test.

\section{Results}

Out of 994 samples, $10.9 \%$ did not access to mobile phones. About $62.7 \%$ of samples had smart phones and internet access via mobile phones. Regarding the use of social networks, $129(13.7 \%)$ of the total research samples were mobile Internet users and $62.7 \%$ of all research samples had access to other services such as Viber, WhatsApp and Instagram. Out of total samples, $270(28.6 \%)$ people sent less than 10 messages daily, while 182 (19.3\%) sent more than 50 messages per day. In the last six months, $34.7 \%$ of samples had sent sext and $15.7 \%$ had received sext.

In case of receiving sext, $663(77.8 \%)$ people said that they quickly deleted the message, $54(6.3 \%)$ forwarded it to others, and 135 (15.8\%) of them saved and sometimes saw it. There was an association between students' gender with receiving $(\mathrm{P}<0.0001)$ and sending $(\mathrm{P}<0.0001)$ sext, so that girls sent and received sexts more than boys. There was no significant statistical difference between the four types of schools regarding students' sexting, although students in technical schools were more likely to do sexting than three other types of schools. There was a statistical significant relationship between sending $(\mathrm{P}<0.0001)$ and receiving sext $(\mathrm{P}<0.0001)$ and the total number of texting per day.

\section{Discussion}

In the present study, the majority of Rafsanjan high school students had access to mobile phones and just $10.9 \%$ had not. This amount of access to mobile phones is much higher than students from the United States and equates to almost all other Iranian reported cases. About three-fourths of the entire research units could use other social networks through mobile phones, which confirms that most students have smart phones, while this proportion is $23 \%$ among American students. It is clear that increasing access to social networks in the form of a mobile phone, which is usually a personal device and is subject to little parental control, can be potentially harmful to the cyber-security of children and young people.

That is why the access to smart phones among children and teens is limited in many countries, and unfortunately, having this device among many of our teens in our country has become a logical need or demand. About one-fifth of the samples sent more than 50 messages per day. Particularly, as the number of messages increases every day, the likelihood of sexting increases; this high volume of text messaging among students should be considered. More than a third of the students reported having sexting in the last six months.

It should be noted that sexting in previous investigations is directly related to the sexual activity among adolescents. Therefore, this high prevalence of sexting can represent a significant amount of sexual activity among adolescents. Studies show that these interactions in this age of ignorance and impotence of adolescents can expose them to problems such as sexually transmitted diseases specially HIV/AIDS as well as unwanted pregnancy and social and behavioral problems and reveals the need for parents supervision and plan for parenting education. 


\section{Ethical Considerations}

Compliance with ethical guidelines

This study was approved by the Ethics Committee of Rafsanjan University of Medical Sciences (code: 93590). Necessary permissions were obtained from the Education Center of Rafsanjan. Also consent was obtained from students.

Funding

This study received financial support from Rafsanjan University of Medical Sciences. It is an approved research project.

Authors contributions

All authors contributed in preparing this article.

\section{Conflict of interest}

The authors declared no conflict of interest.

Acknowledgements

Authors would like to thank authorities of study high schools and parents of students for their valuable cooperation. 


\title{
ميزان دسترسى به تلفن همراه و شيوع استفاده از آن براي تبادل مفاهيم جنسى در بين دانش آموزان دبيرستانى شهر رفسنجان، سال عوسئ
}

\author{
زهره قرشى' • • •مرضيه لرىيور' •، سيده مريم لطفىيور رفسنجانى '
}

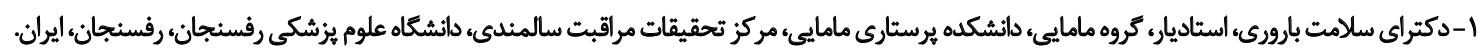

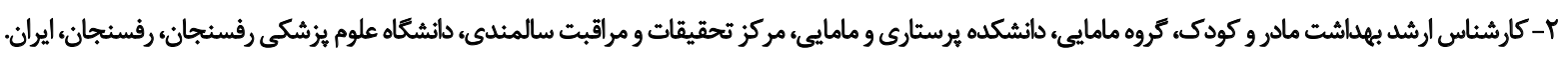

\section{حكيد}

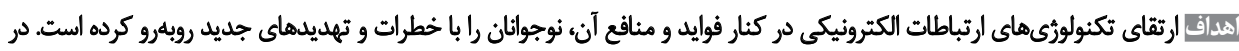

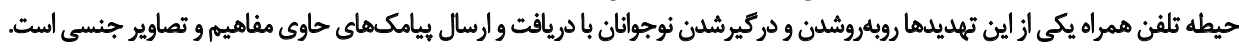

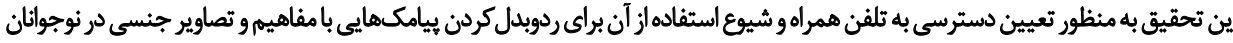

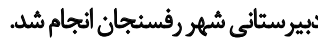

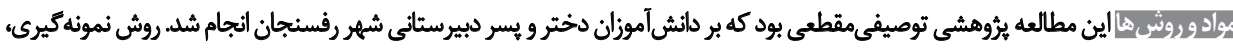

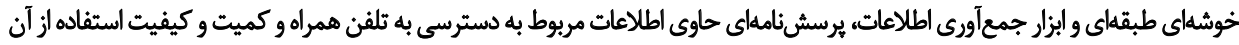

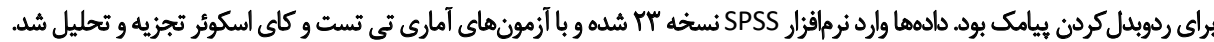

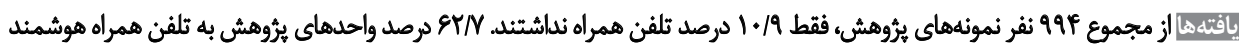

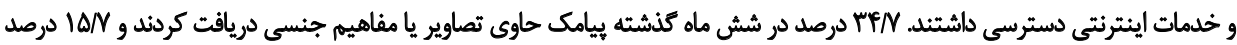

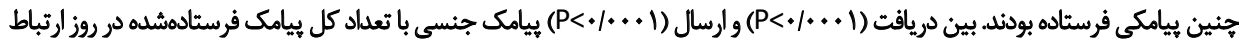

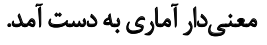

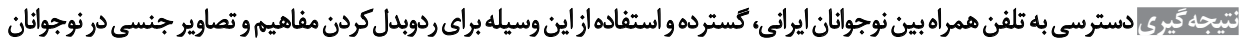

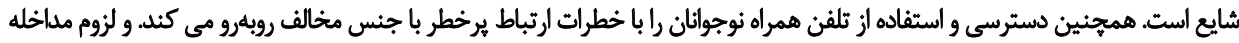

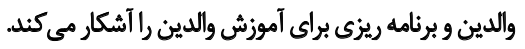

تاريخ دريافت: ب شهريور

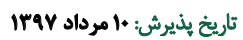

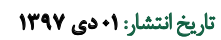

كليدوازهها:

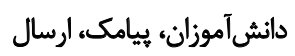
مفاهيم و تصاوير جنسي

دسته آسيبهاى ناشى از ثلفن همراه قرار مي كرفتند [Y].

dates

يك تحقيق نشان مى دهد در ميان نوجوانان با تا با باساله

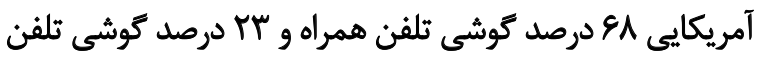

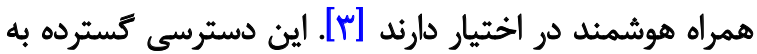

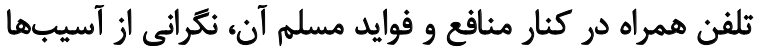

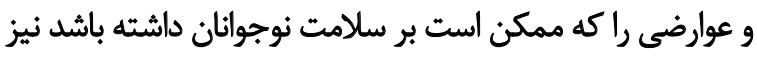

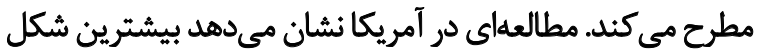

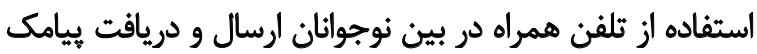

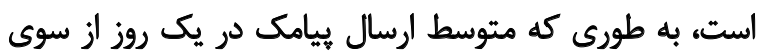

نوجوانان به •و مورد ميرسد متوسط ارسال]

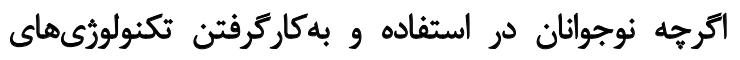

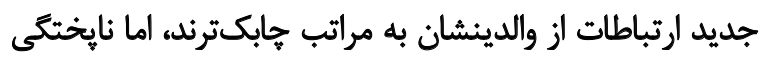

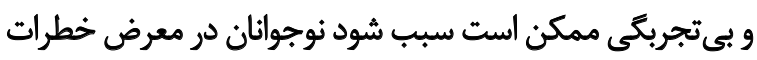

فروزه ارتقاى تكنولوزيىهاى ارتباطات الكترونيكى مثل تبادل

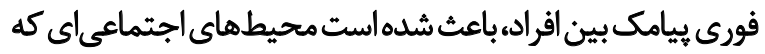

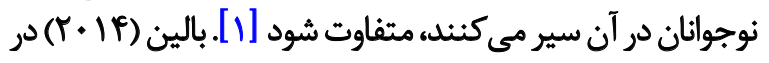

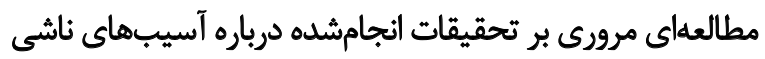

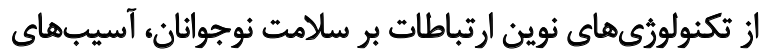

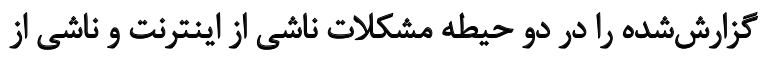

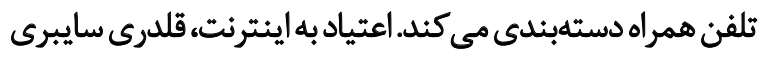

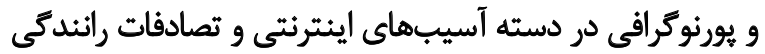

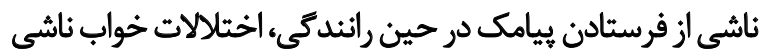

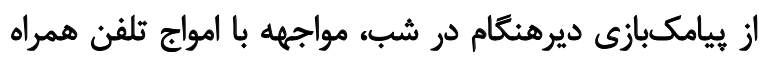

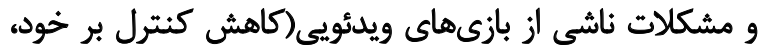

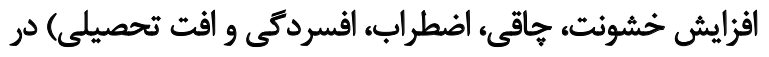

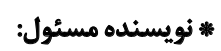

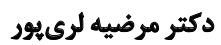

$$
\begin{aligned}
& \text { نشانيى: رفسنجان، دانشكاه علوم يزشكى رفسنجان، دانشكده يرستارى مامايى، كروه مامايى. }
\end{aligned}
$$

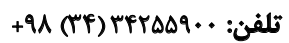

$$
\begin{aligned}
& \text { يست الكترونيكى: marzeyehloripoor@yahoo.com }
\end{aligned}
$$


انتخاب نمونهها خوشهايىطبقهاى و انتخاب خوشهها و طبقهها

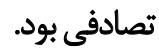

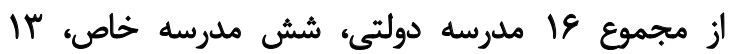

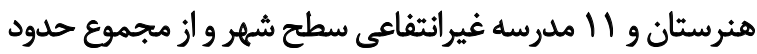

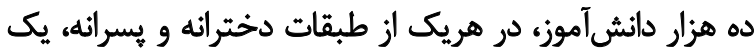

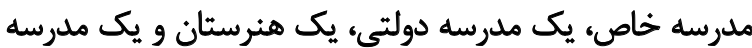

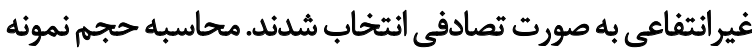

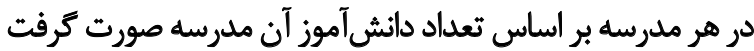
و به ميزان • إدرصد كل تعداد دانش آموزان مدرسه انجام شد.

تكميل يرسشنامهها به وسيله واحدهاى يرؤهش انجام شد.

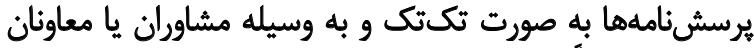

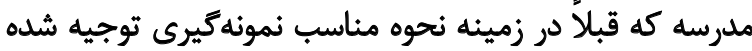

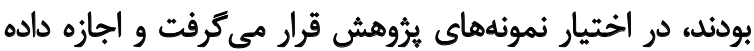

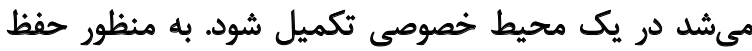

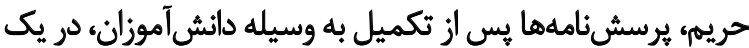

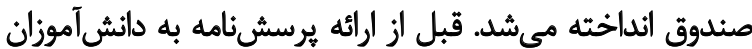

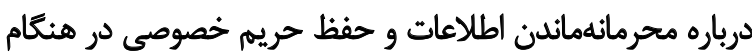

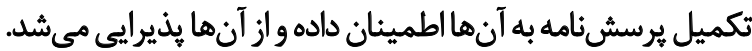

ابزار جمعآورى دادهها يك يرسشنامه يثروهشكرساخته بود كه

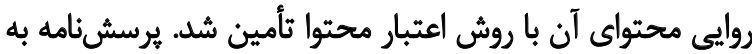

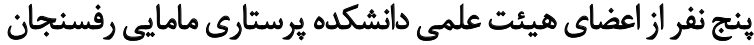

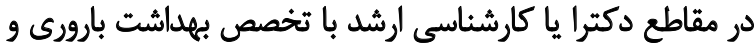

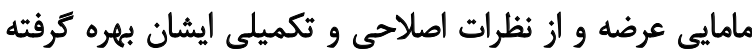

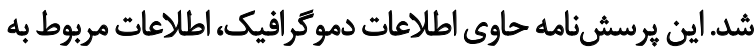

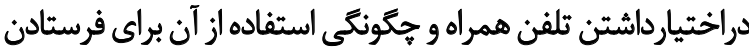

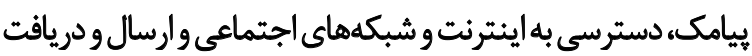

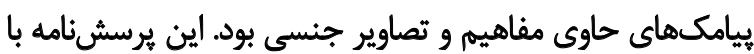

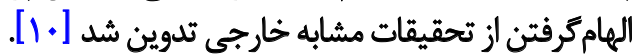
يايايى يرسش نامه به روش آلفاي كرونباخ محاسبه نشد كه علت

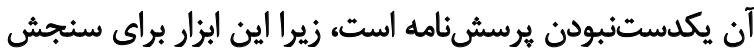

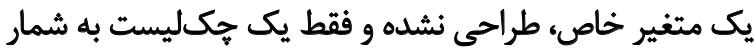

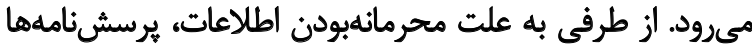

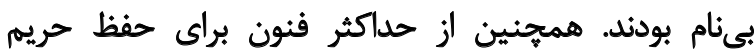

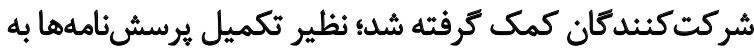

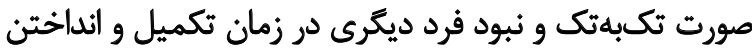

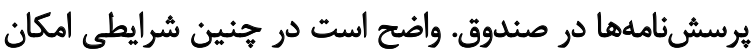
آزمون بازآزمون نيز ميسر نبوده است.

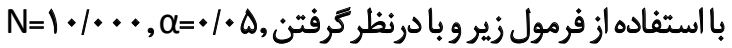

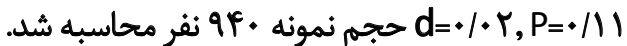
$N Z 2[1-\alpha / 2 P(1-P) / d(N-1)+Z 2] 1-\alpha / 2 P(1-P)$

با توجه به امكان ريزش نمونهها به علت تكميل ناقص
خاص و آسيبهاى جدى ناشى از اين تكنولوزيىهاى نوين قرار

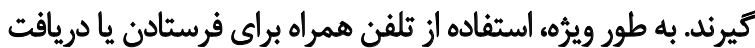

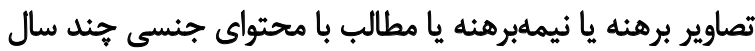

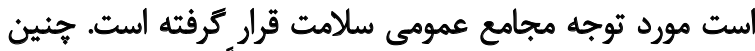

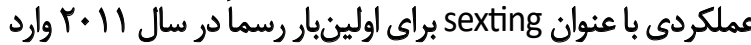
زبان انظليسى و در فرهنگ لغت آكسفورد ثبت شد [هـ].

در ايران نيز ميزان استفاده از تلفن همراه در بين نوجوانان

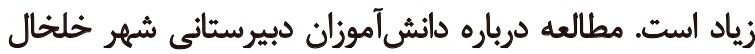

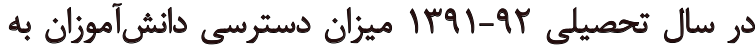

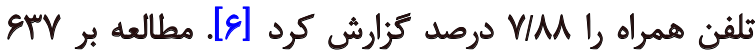

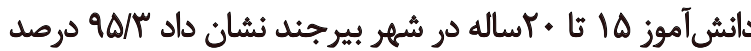

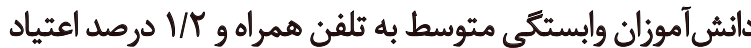

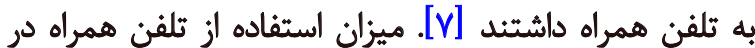

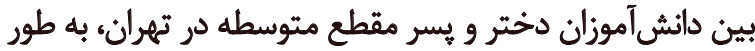

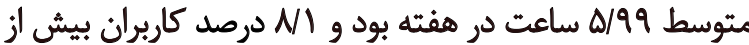

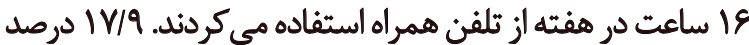

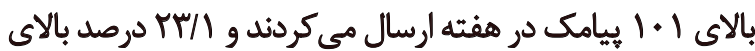

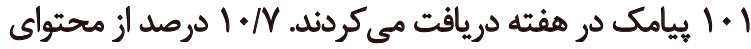

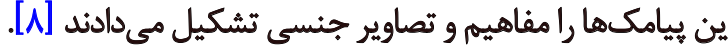
تحقيقات نشان مى دهد نوجواناني كه مفاهيم و تصاوير جنسى

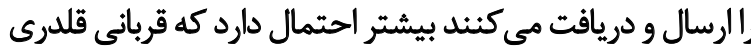

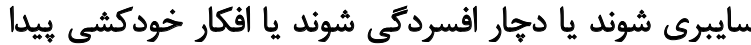

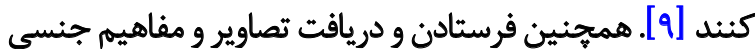

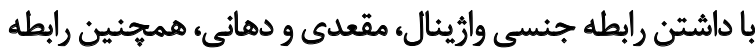
جنسى محافظتشده ارتباط داشته است [ـ [1]

با درنظركرفتن اين حجم بالاي استفاده از تلفن همراه و بلويرُه

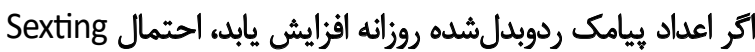

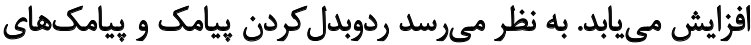

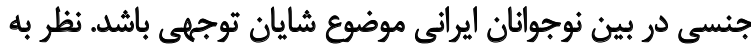

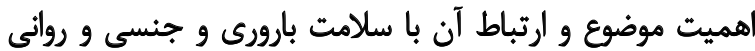

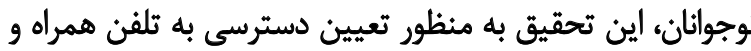

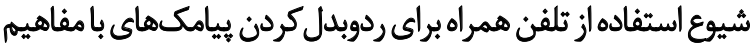

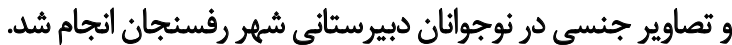

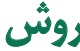

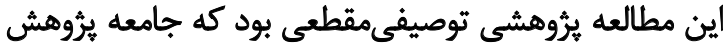

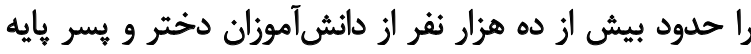

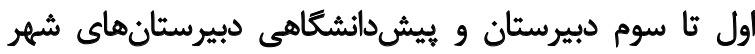

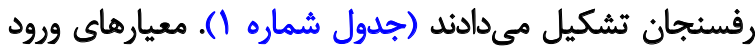

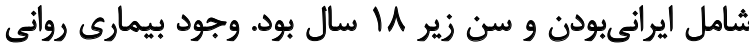

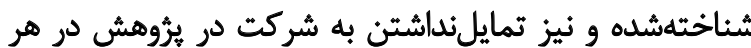

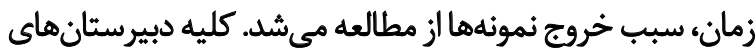
شهر رفسنجان به عنوان محيط يُوهش در نظر ترفته شد. روش 


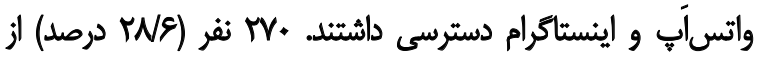

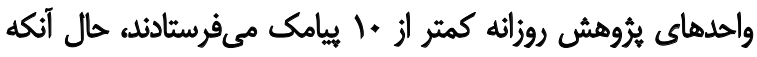

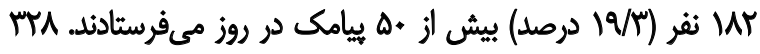

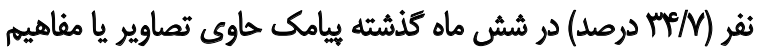

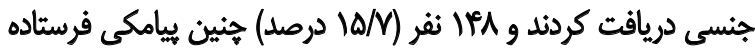

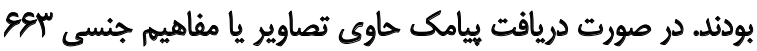

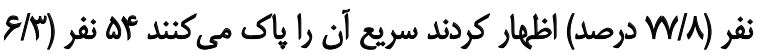

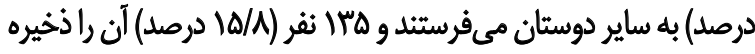
مى كثند و كاهى مى بييند.

دسترسى به ثلفن همراه با ميانگين سن واحدهاى يُروهش

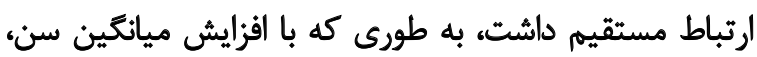

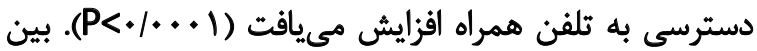

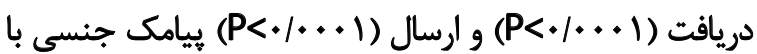

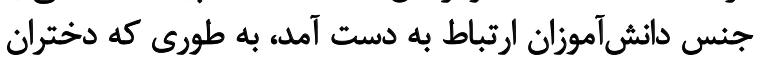

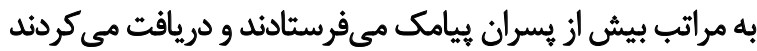

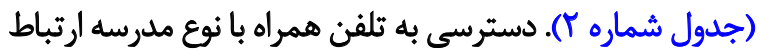

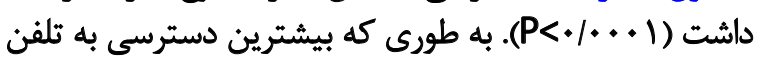

يرسشنامهها تعداد نمونه هزار نفر در نظر كرفته شد كه نهايتاً

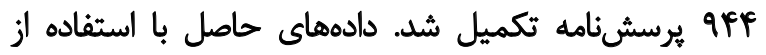

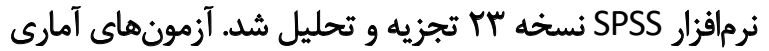
استفادهشه شامل آزمونهاى تى تست، كاى نسئ اسكوئر بود.

ياقتهها

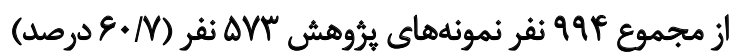

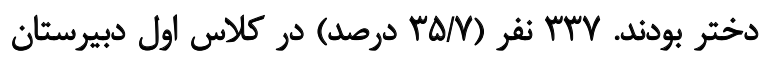

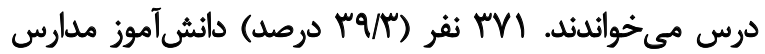

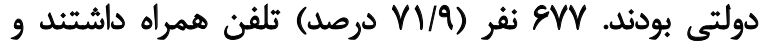

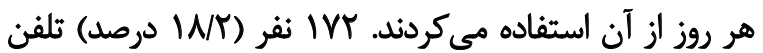

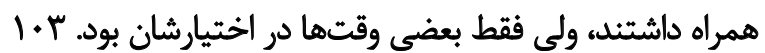

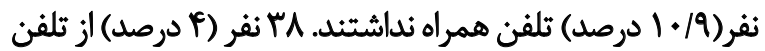
همراه ساير اعضاى خانواده استفاده مى كردند. نماهن.

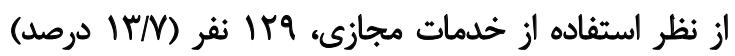

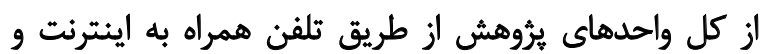

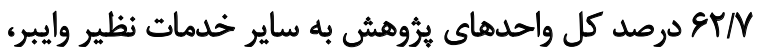

جدول ا. توزيع فراوانى نسبى نمونههاي يرؤهش برحسب متغيرهاى جمعيتشناختى

\begin{tabular}{|c|c|}
\hline تعداد (درصد) & متغير \\
\hline$m r(r q / f)$ & يسر \\
\hline$\Delta V r(\varepsilon \cdot 18)$ & 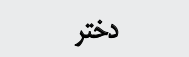 \\
\hline$M V(r \Delta / q)$ & بايه اول دييرستان \\
\hline$M M(T r / A)$ & جايه دوم دييرستان \\
\hline $\operatorname{rgT}(\Upsilon \wedge)$ & هايه سوم ديبرستان \\
\hline$\| F(I Y / T)$ & يبيش دانشكاهى \\
\hline $\operatorname{rAT}(\mathrm{TE} / \mathrm{q})$ & 10 10 \\
\hline $\operatorname{rer}(r+/)$ & عا ساله \\
\hline$r m(r q)$ & \\
\hline$|r|(1 r / q)$ & 1A ساله \\
\hline
\end{tabular}

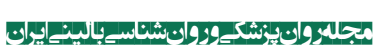

جدول ب. توزيع جنسيتى ارسال و دريافت ييامكهاى با مفاهيم جنسى در بين واحدهاى يُروهش

\begin{tabular}{|c|c|c|c|c|}
\hline \multicolumn{2}{|c|}{ دريافت مغاهيه و تصاوير جنسى (درصد) } & \multicolumn{2}{|c|}{ فرستادن مفاهيم و تصاوير جنسى (درصد) } & \multirow{2}{*}{ جنسيت } \\
\hline خير & بله & خير & بله & \\
\hline$r \cdot q(\Delta H / T)$ & $\operatorname{rar}(V \& / \Lambda)$ & PrN(AV/\&) & $\operatorname{Irr}(A T / F)$ & دختران \\
\hline $\operatorname{Mr}(\kappa \varepsilon / \mathcal{A})$ & $V g(T / / T)$ & $\pi T(F T / F)$ & $r q(I V / g)$ & يسران \\
\hline$\Delta A)(1 \cdots)$ & MTA(1..) & $v 8 \cdot(1 .)$. & IFA(1..) & جمع \\
\hline
\end{tabular}

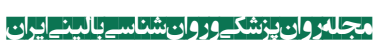


دوم دبيرستان را بررسى نكردهاند. بايد توجه كرد دريافت و ارسال

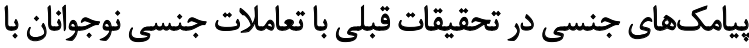

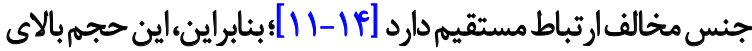

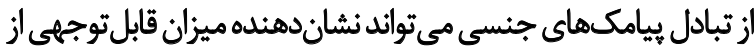
ارتباطات و تعاملات جنسى نوجوانان با جنس مخالف باشند.

مطالعات نشان ميى دهند اين تعاملات در اين سن كه سن نآنآهى

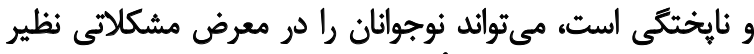

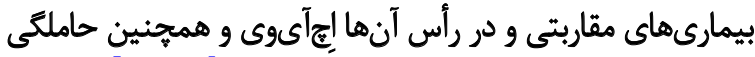

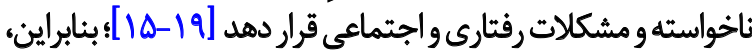

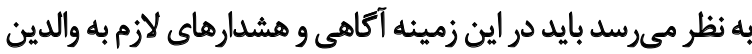

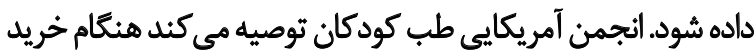

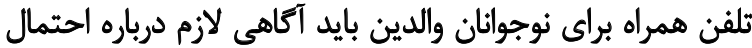

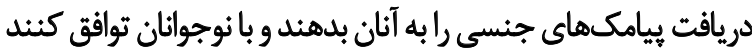

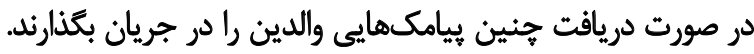

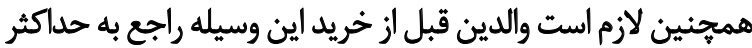

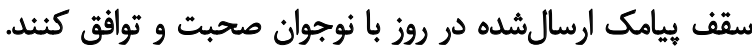

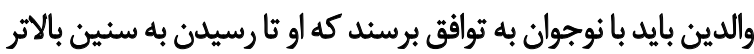

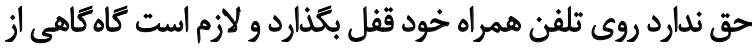

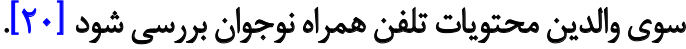
در اين تحقيق از اداره كل آموزش و يرورش شهرستان مجوز

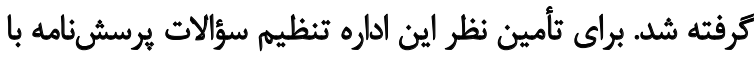

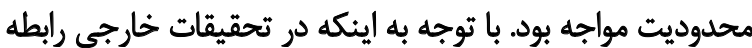

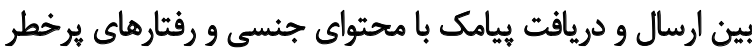

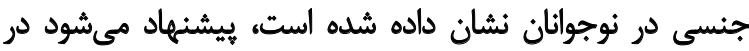
تحقيقات ديخًر به اين موضوع برداخته شود.

\section{نتيجهئميرى}

يافتهاي يرؤهش نشان داد دسترسى به تلفن همراه در بين

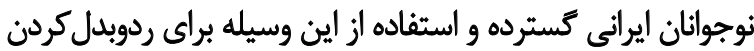

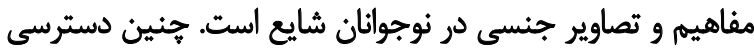

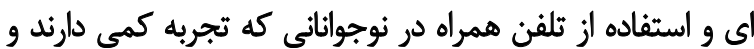

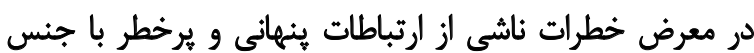

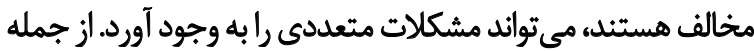

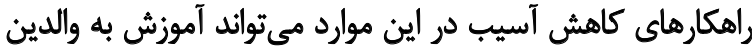

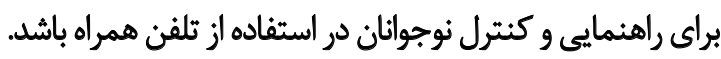

ملاحظات اخلاقي

\section{يبيروى از اصول اخلاث يثوهش}

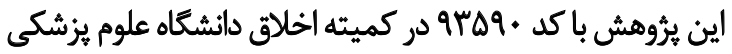

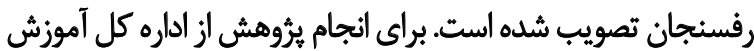

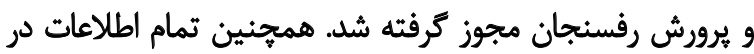
جلسات خصوصى و با رضايت از دانش آموزان كرفته و يرسين نمام اطناعهات درا
همراه در مدارس شاهد و تيزهوشان و كمترين دسترسى در

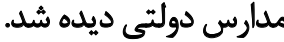

بين ردوبدلكردن ئيامك جنسى و نوع مدرسه اختلاف

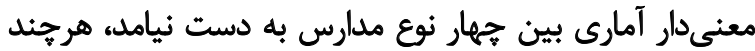

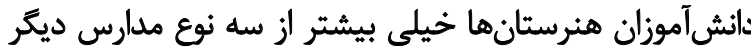

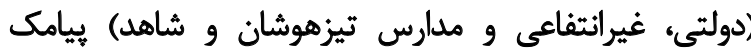

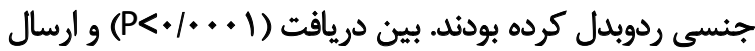

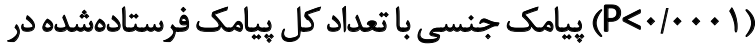

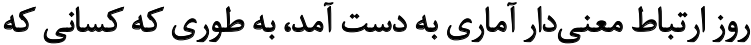

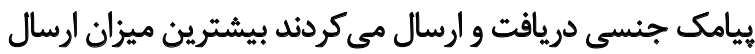

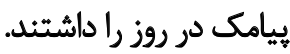

بحث

در اين تحقيق بيشتر دانشآموزان دبيرستانهاى شهرستان

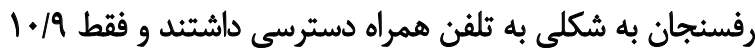

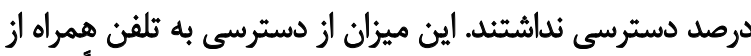

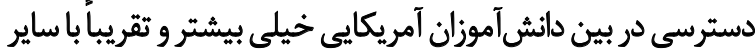

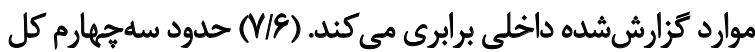

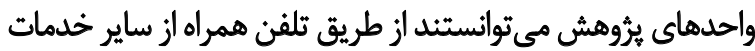

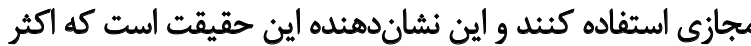

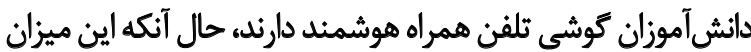

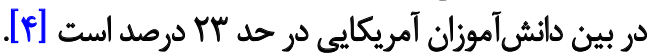
واضح است افزايش دسترسى به خدمات مجازى در قالب يك

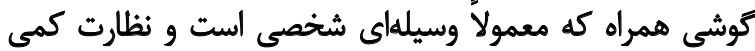

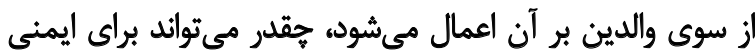

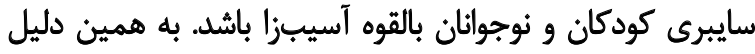

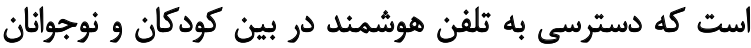

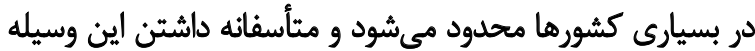

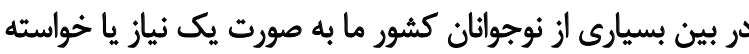

$$
\text { منطقى درآمده است. }
$$

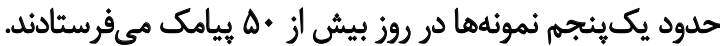

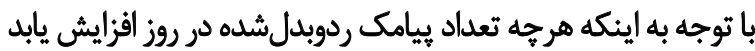

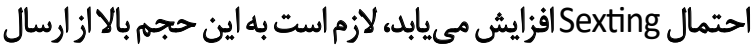

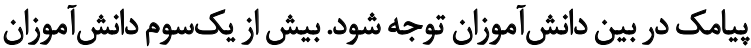

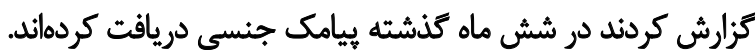

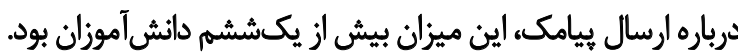
از لحاظ تأثير جنسيت بر رفتارهاى ييامكى دانش آموزان، ارسال و

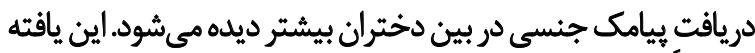

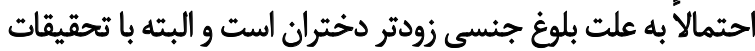

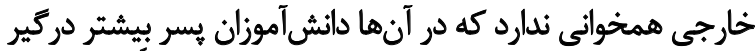

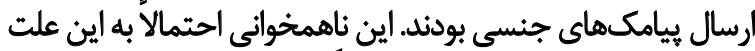

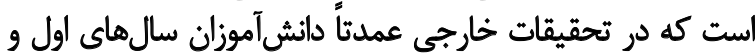


در صندوق انداخته شُد.

$$
\text { مامى مالى }
$$

دانشكاه علوم يزشكى رفسنجان هزينههاي اين يُّوهش را تأمين

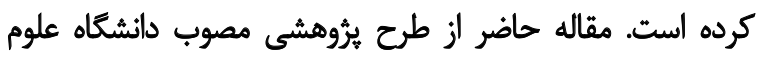

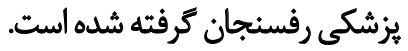

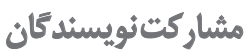

تمام نويسئدكان در آمادهازى اين مقاله مشاركت داشتهاند.

$$
\text { ثارض مثأق }
$$

بنابر اظهارنويسندكان، اين مقاله هيج تعارض منافعى نداشته است.

$$
\text { سباسكَزارى }
$$

بدينوسيله از مشاوران، معاونان و اولياى مدارس سياسكزاريم كه نهايت همكارى را در جمعآورى اطلاعات معات معتبر و ايجاد حريم معان خصوصي داشتيتند. 


\section{References}

[1] Houck C, Barker D, Rizzo C, Hancock E, Norton A, Brown L. Sexting and sexual behavior in at-risk adolescents. Pediatrics. 2014; 133(2):276-82. [DOI:10.1542/peds.2013-1157] [PMID] [PMCID]

[2] Bailin A, Milanaik R, Adesman A. Health implications of new age technologies for adolescents: A review of the research. Current Opinion in Pediatrics. 2014; 26(5):605-19. [DOI:10.1097/ MOP.0000000000000140] [PMID]

[3] Madden M, Lenhart A, Duggan M, Cortesi S, Gasser U. Teenagers and technology 2013 [Internet]. 2013 [Updated 2013 March 13]. Available from: http://www.pewinternet.org/2013/03/13/ teens-and-technology-2013/

[4] Lenhart A. Teens, smart phones \& texting [Internet]. 2012 [Updated 2012 March 19]. Available from: http:/ / www.pewinternet. org/2012/03/19/teens-smartphones-texting/

[5] Couts A. Sextin, retweet and woot added to the Oxford English Dictionary [Internet]. 2011 [Updated 2011 August 18]. Available from: https://www.digitaltrends.com/web/sexting-retweetand-woot-added-to-the-oxford-english-dictionary/

[6] Atadokht A, hamidifa V, mohammadi E. [Over-use and type of mobile phone users in high school students and its relationship with academic performance and achievement motivation (Persian)]. School Psychology Digest. 2014; 3(2):122-36.

[7] Khazaie T, Saadatjoo A, Dormohamadi S, Soleimani M, Toosinia M, Mullah Hassan Zadeh F. [Prevalence of mobile dependency and adolescence aggression (Persian)]. Journal of Birjand University of Medical Sciences. 2013; 19(4):430-8.

[8] Moidfar S, Ganji A. [An analysis of the various functions of the mobile phone practiced by high school male and female students in Tehran (Persian)]. Global Media Journal. 2009; 8(4):79-103.

[9] Medrano JLJ, Lopez Rosales F, Gámez-Guadix M. Assessing the links of sexting, cybervictimization, depression, and suicidal ideation among university students. Archives of Suicide Research. 2018; 22(1):153-64. [DOI:10.1080/13811118.2017.1304304] [PMID]

[10] Rice E, Craddock J, Hemler M, Rusow J, Plant A, Montoya J, et al. Associations between sexting behaviors and sexual behaviors among mobile phone-owning teens in Los Angeles. Child Development. 2018; 89(1):110-7. [DOI:10.1111/cdev.12837] [PMID]

[11] Temple JR, Paul JA, van den Berg P, Le VD, McElhany A, Temple BW. Teen sexting and its association with sexual behaviors. Archives of Pediatrics and Adolescent Medicine. 2012; 166(9):82833. [DOI:10.1001/archpediatrics.2012.835] [PMID] [PMCID]

[12] Temple JR, Choi H. Longitudinal association between teen sexting and sexual behavior. Pediatrics. 2014; 134(5):e1287-92. [DOI:10.1542/peds.2014-1974] [PMID] [PMCID]

[13] Dir AL, Cyders MA. Risks, risk factors, and outcomes associated with phone and internet sexting among university students in the United States. Archives of Sexual Behavior. 2015; 44(6):167584. [DOI:10.1007/s10508-014-0370-7] [PMID]

[14] Smith LW, Liu B, Degenhardt L, Richters J, Patton G, Wand $\mathrm{H}$, et al. Is sexual content in new media linked to sexual risk behaviour in young people? A systematic review and metaanalysis. Sexual Health. 2016; 13(6):501-15. [DOI:10.1071/ SH16037] [PMCID]
[15] Strassberg DS, Cann D, Velarde V. Sexting by high school students. Archives of Sexual Behavior. 2017; 46(6):1667-72 [DOI:10.1007/s10508-016-0926-9] [PMID]

[16] Kalamar AM, Bayer AM, Hindin MJ. Interventions to prevent sexually transmitted infections, including HIV, among young people in low- and middle-income countries: A systematic review of the published and gray literature. Journal of Adolescent Health. 2016; 59(3):S22-31. [DOI:10.1016/j.jadohealth.2016.05.020]

[17] Kahn NF, Halpern CT. Associations between patterns of sexual initiation, sexual partnering, and sexual health outcomes from adolescence to early adulthood. Archives of Sexual Behavior. 2018; 47(6):1791-810.[DOI:10.1007/s10508-018-1176-9]

[18] Mahat G, Scoloveno MA. Effectiveness of adolescent peer education programs on reducing HIV/STI risk: An integrated review. Research and Theory for Nursing Practice. 2018; 32(2):168 98. [DOI:10.1891/1541-6577.32.2.168] [PMID]

[19] Sperhacke RD, da Motta LR, Kato SK, Vanni AC, Paganella $\mathrm{MP}$, de Oliveira MC, et al. HIV prevalence and sexual behavior among young male conscripts in the Brazilian army, 2016. Medicine. 2018; 97(1S):S25-S31. [DOI:10.1097/MD.0000000000009014] [PMID] [PMCID]

[20] American Academy of Pediatrics. Talking to Kids and Teens About Social Media and Sexting -Tips from the American Academy of Pediatrics [Internet]. 2013 [Updated 2013 May 31]. Available from: https://www.aap.org/en-us/about-the-aap/ aap-press-room/news-features-and-safety-tips/Pages/Talkingto-Kids-and-Teens-About-Social-Media-and-Sexting.aspx 
This Page Intentionally Left Blank 